HERIOT

原国 WATT

UNIVERSITY

Heriot-Watt University

Research Gateway

\title{
Increasing circular synthetic aperture sonar resolution via adapted wave atoms deconvolution
}

\section{Citation for published version:}

Pailhas, Y, Petillot, Y \& Mulgrew, B 2017, 'Increasing circular synthetic aperture sonar resolution via adapted wave atoms deconvolution', Journal of the Acoustical Society of America, vol. 141, no. 4, pp. 26232632. https://doi.org/10.1121/1.4979807

\section{Digital Object Identifier (DOI):}

$10.1121 / 1.4979807$

\section{Link:}

Link to publication record in Heriot-Watt Research Portal

\section{Document Version:}

Publisher's PDF, also known as Version of record

\section{Published In:}

Journal of the Acoustical Society of America

\section{Publisher Rights Statement:}

Copyright (year) Acoustical Society of America. This article may be downloaded for personal use only. Any other use requires prior permission of the author and the Acoustical Society of America.

The following article appeared in The Journal of the Acoustical Society of America 141, 2623 (2017) and may be found at http://asa.scitation.org/doi/10.1121/1.4979807

\section{General rights}

Copyright for the publications made accessible via Heriot-Watt Research Portal is retained by the author(s) and / or other copyright owners and it is a condition of accessing these publications that users recognise and abide by the legal requirements associated with these rights.

\section{Take down policy}

Heriot-Watt University has made every reasonable effort to ensure that the content in Heriot-Watt Research Portal complies with UK legislation. If you believe that the public display of this file breaches copyright please contact open.access@hw.ac.uk providing details, and we will remove access to the work immediately and investigate your claim. 


\title{
Increasing circular synthetic aperture sonar resolution via adapted wave atoms deconvolution
}

\author{
Yan Pailhas, ${ }^{1, a)}$ Yvan Petillot, ${ }^{1}$ and Bernard Mulgrew ${ }^{2}$ \\ ${ }^{1}$ School of Engineering and Physical Sciences, Heriot-Watt University, Edinburgh, United Kingdom \\ ${ }^{2}$ School of Engineering, University of Edinburgh, Edinburgh, United Kingdom
}

(Received 19 August 2016; revised 22 March 2017; accepted 24 March 2017; published online 13 April 2017)

\begin{abstract}
Circular Synthetic Aperture Sonar (CSAS) processing computes coherently Synthetic Aperture Sonar (SAS) data acquired along a circular trajectory. This approach has a number of advantages, in particular it maximises the aperture length of a SAS system, producing very high resolution sonar images. CSAS image reconstruction using back-projection algorithms, however, introduces a dissymmetry in the impulse response, as the imaged point moves away from the centre of the acquisition circle. This paper proposes a sampling scheme for the CSAS image reconstruction which allows every point, within the full field of view of the system, to be considered as the centre of a virtual CSAS acquisition scheme. As a direct consequence of using the proposed resampling scheme, the point spread function (PSF) is uniform for the full CSAS image. Closed form solutions for the CSAS PSF are derived analytically, both in the image and the Fourier domain. The thorough knowledge of the PSF leads naturally to the proposed adapted atom waves basis for CSAS image decomposition. The atom wave deconvolution is successfully applied to simulated data, increasing the image resolution by reducing the PSF energy leakage. (C) 2017 Acoustical Society of America.

[http://dx.doi.org/10.1121/1.4979807]
\end{abstract}

$[\mathrm{ZHM}]$

Pages: 2623-2632

\section{INTRODUCTION}

Since Bellettini and Pinto's paper in 2002, ${ }^{1}$ which solves the problem of micro-navigation, several SAS (Synthetic Aperture Sonar) systems, including commercial systems, have been developed. ${ }^{2-5}$ The various advantages of these systems, such as very high resolution sonar images, constant resolution over range, or large swath, have made SAS systems the tool of choice for large areas survey and/or MCM (Mine-Counter Measure) operations. ${ }^{6,7}$ The Automatic Target Recognition algorithm performances have greatly improved ${ }^{8-10}$ with the use of SAS high resolution images. It has became clear however that multiple views are necessary to reduce the false alarm rate to an acceptable level. ${ }^{11,12}$ Different reacquisition strategies have been explored including repeat pass, or daisy lawnmower patterns. An interesting target reacquisition pattern consists in performing a circle around the area of interest. This approach has several advantages over the daisy lawnmower pattern, e.g., the sensor acquires continuously all the $360^{\circ}$ views of the target of interest. In addition the circular reacquisition pattern is also relatively efficient in terms of acquisition time ${ }^{13,14}$ For synthetic aperture systems, however, the use of a circular trajectory raises the interesting problem of image reconstruction.

Circular synthetic aperture radar (CSAR) has been investigated for several years, ${ }^{15-17}$ and image reconstruction algorithms have been proposed including tomographic or back-projection reconstruction. CSAR has been applied to 3D (three-dimensional) extraction, ${ }^{15,18}$ ground moving target

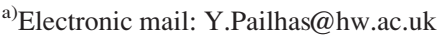

detection, ${ }^{19}$ interferometric multipass,${ }^{20}$ or ground penetrating applications. ${ }^{15}$ Recently, Circular SAS (also known as CSAS) has also gained some interest from the MCM community. Ferguson and Wyber ${ }^{21}$ and Hansen et al. ${ }^{22}$ demonstrated the feasibility of fully integrating the synthetic antenna along a circular path in the late 2000s: early CSAS images are presented ${ }^{21}$ showing the capabilities of such approach. Ameliorations in the focusing process have been proposed, for example, with controlled focus in Ref. 23, or more recently by Marston et al. in Ref. 24 where the authors correct the focus aberration due to the accumulation of the phase error using phase correction modelled by generalised cones. CSAS processing also opens new perspectives, e.g., repeat circular path can recover the 3D image of an area of interest. $^{25}$

This paper proposes a general theoretical study on CSAS and on the CSAS image formation in particular. If the feasibility of such systems has been proven, the derivation of CSAS processing, however, has not been fully developed. Such theoretical derivations can greatly help the development of new filters whether for denoising, deconvolution, or compression. The main contributions of this paper are:

- A new sampling scheme is proposed for circular acquisition using the SAS system. By sampling properly along the trajectory, every point within the field of view of the system can be seen as the centre of a virtual CSAS.

- Theoretical closed form solutions for the impulse response of a CSAS system are computed in the spatial domain and in the Fourier domain. An approximated expression of the point spread function (PSF) energy diffusion is also 
proposed, and leads to redefine CSAS resolution based on the PSF energy leakage.

- Thanks to the analytical expression of the PSF, an adapted atom wave basis is proposed. Decomposition in this basis concentrates the CSAS image energy which facilitates the deconvolution process and increases the CSAS resolution.

The paper is organised as follows: Section II A describes the CSAS problem and proposes a new sampling scheme to process CSAS data. In Sec. II B, the analytical solutions are given for the matched filter response (Sec. II C) and the CSAS processing PSF. A discussion on the resolution of CSAS systems is proposed in Sec. II D, in particular the energy leakage of the PSF is discussed. The analytical solution of the PSF in the Fourier domain is computed in Sec. III A, which leads to the design of the wave atoms for waveform adapted deconvolution. Simulated results and discussions are presented in Sec. III B.

\section{CSAS PROCESSING}

This section defines the CSAS geometry and calculates analytically the theoretical PSF expression for circular synthetic aperture systems. The PSF is then linked to the resolution capabilities of the CSAS system. The rest of the paper will use the notations and nomenclature defined in Table I.

\section{A. Configuration}

A SAS system $\mathbf{S}$ describes a circular trajectory $\mathcal{C}$ centred on the point $\mathbf{O}$ and with a radius $\mathrm{R}$ as seen in Fig. 1(a). At time $\tau$, the system $\mathbf{S}$, considered static during the acquisition, sends the pulse $s(t)$ and records the corresponding echo $s^{\tau}(t)$. Note that $t$ is often referred to as the fast time, while $\tau$ is referred to as the slow time.

The full view area is defined as the area where every point is seen by the sensor $\mathbf{S}$ during all its trajectory on $\mathcal{C}$. As pictured in Fig. 1(a), the full view area, highlighted in blue, is a circle whose radius $r$ is

$$
r=\mathrm{R} \sin (\varphi / 2),
$$

where $\varphi$ is the beamwidth of the SAS system. Let $\mathbf{O}^{\prime}$ be a point within the full view area. $\mathbf{O O}^{\prime}=\alpha \mathrm{R}$ where $\alpha \in[0, \sin (\varphi / 2)]$. Let $\theta^{\prime}$ be the view angle of the point $\mathbf{O}^{\prime}$ from the sensor and $\theta$ the corresponding view angle of the centre $\boldsymbol{O}$. The two angles $\theta$ and $\theta^{\prime}$, defined in $[-\pi, \pi]$, are linked through the following two equations:

$$
\theta^{\prime}=h(\theta)=\operatorname{sgn}(\theta) \cos ^{-1}\left(\frac{\cos \theta-\alpha}{\sqrt{1+\alpha^{2}-2 \alpha \cos \theta}}\right),
$$

TABLE I. Nomenclature.

\begin{tabular}{lcc}
\hline \hline Notations & Definition & Units \\
\hline$c$ & sound speed & $\mathrm{m} \mathrm{s}^{-1}$ \\
$f_{0}$ & pulse centre frequency & $\mathrm{Hz}$ \\
$\Delta f$ & Bandwidth & $\mathrm{Hz}$ \\
$T$ & pulse length & $\mathrm{s}$ \\
$\sigma$ & Gaussian window temporal width & - \\
\hline \hline
\end{tabular}

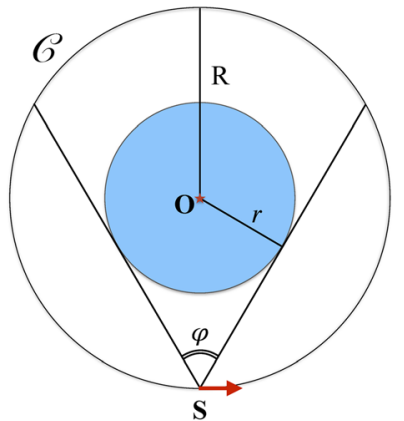

(a)

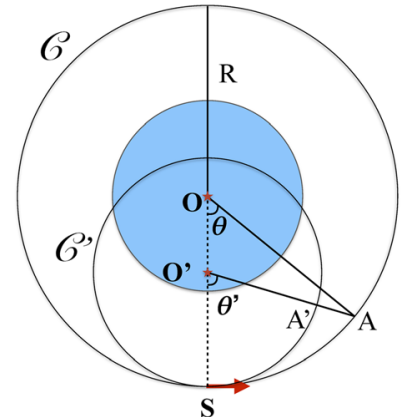

(b)
FIG. 1. (Color online) CSAS configuration: $\mathbf{S}$ location of the SAS system following the circular trajectory $\mathcal{C}$. (a) Graphic representation of the full view area (highlighted in blue), and (b) isomorphism between the real $(\mathcal{C})$ and virtual trajectory $\left(\mathcal{C}^{\prime}\right)$.

$$
\begin{aligned}
\theta & =h^{-1}\left(\theta^{\prime}\right) \\
& =\operatorname{sgn}\left(\theta^{\prime}\right) \cos ^{-1}\left(\alpha \sin ^{2} \theta^{\prime}+\cos \theta^{\prime} \sqrt{1-\alpha^{2} \sin ^{2} \theta^{\prime}}\right) .
\end{aligned}
$$

The CSAS response of $\mathbf{O}^{\prime}, I\left(\mathbf{O}^{\prime}\right)$, is traditionally computed by integrating all the matched filter echo responses acquired along the SAS trajectory $\mathcal{C}$ and can be mathematically described by Eq. (4),

$$
I\left(\mathbf{O}^{\prime}\right)=\int_{\mathcal{C}} s_{f}^{\tau}\left(t_{\mathbf{O}^{\prime}}\right) \mathrm{d} \tau
$$

where $s_{f}^{\tau}(t)$ is the match filtered response of the echo $s^{\tau}(t)$ received at time $\tau, t_{\mathbf{O}^{\prime}}=\mathbf{S O}^{\prime} / c$ is wave travel time between $\mathbf{S}$ and $\mathbf{O}^{\prime}$ and $c$ the sound speed. Note that Eq. (4) is in essence a generalized time domain back propagation integration along the trajectory $\mathcal{C}$. It is important to note that this formulation does not assume plane wave propagation. Other formulations of the CSAS response using the plane wave propagation assumption have been proposed in the past. $^{21,26}$

Along the trajectory $\mathcal{C}$, the sampling is performed at an $\lambda / 2$ interval, where $\lambda$ is the pulse wavelength. Because the relation between $\theta$ and $\theta^{\prime}$ is not linear, integrating along $\mathcal{C}$ to estimate $\mathbf{O}^{\prime}$ may result in some discrepancy due to nonuniform sampling. The sample density as a function of the angle of integration is in fact uneven if $\mathbf{O}^{\prime}$ is far from the centre $\mathbf{O}$. As a consequence, the PSF is not uniform within the full view area. The proposed integration method and resampling of Eq. (4) below aims to tackle precisely the non-uniformity of the PSF due to the uneven angular sampling of the CSAS processing. Results and PSF formula in Sec. II C are then valid for the full view area and the deconvolution proposed in Sec. III B is greatly simplified.

The integration for $\mathbf{O}^{\prime}$ can be done properly by integrating Eq. (4) along $\mathcal{C}^{\prime}$ at $\lambda / 2$ interval. By putting

$$
\theta_{n}^{\prime}=n \frac{\lambda}{2} \frac{1}{(1-\alpha) R}
$$

into Eq. (3), the integration then is uniformly sampled, at an $\lambda / 2$ interval, along $\mathcal{C}^{\prime}$, 


$$
I\left(\mathbf{O}^{\prime}\right)=\int_{\mathcal{C}^{\prime}} s_{f}^{\tau^{\prime}}(t) \mathrm{d} \tau^{\prime}
$$

To compute Eq. (6), one can note that the sampling acquisition time $\tau$ is intrinsically linked to the view angle $\theta$. As a consequence, integrating $s_{f}^{\tau}$ along the slow time $\tau$ is equivalent to integrating $s_{f}^{\theta}$ along the view angle $\theta$. The effective resampling suggested by Eq. (6) can then be computed numerically as follows:

$$
I\left(\mathbf{O}^{\prime}\right)=2 \pi \sum_{n=1}^{N^{\prime}} s_{f}^{h^{-1}\left(\theta_{n}^{\prime}\right)}\left(t_{\mathbf{O}^{\prime}}\right),
$$

where $\theta_{n}^{\prime}$ is given by Eq. (5) and the function $h^{-1}(\cdot)$ by Eq. (3). There is an obvious dissymmetry between the centred point and the offset points due to the CSAS geometry. The proposed sampling scheme given by Eq. (7) allows every point in the full view area to be interpreted as the centre of a CSAS acquisition. The rest of this paper only focuses on the imaging process of $\mathbf{O}$, centre of $\mathcal{C}$, and its neighbourhood, but the results of Secs. II and III are valid for all the points within the full view area. As previously stated, by using the sampling scheme suggested by Eq. (7), the CSAS PSF, derived in Secs. II C and III A, is uniform over the full view area.

\section{B. Matched filter response}

Assuming that the pulse $s(t)$ sent by the system is a linear frequency modulated chirp, $s(t)$ can then be written as

$$
s(t)=A(t) e^{2 i \pi\left(f_{0}+[\Delta f / 2 T] t\right) t},
$$

where $A(t)$ is the windowing function, $f_{0}$ the centre frequency, $\Delta f$ the bandwidth, and $T$ the pulse duration. $A(t)$ plays an important role in the final SAS image, as will be shown in Sec. II C, and is also subject to various constraints. Note that in this paper $e^{z}$ and $\exp (z)$ are used indiscriminately to refer to the exponential function. $e^{z}$ is used for compactness when the expression $z$ is relatively compact, and $\exp (z)$ is used for better readability when the expression $z$ becomes too complex.

In radar systems, the amplifiers used for pulse transmission are non-linear and the windowing function for radar systems, also known as the constant amplitude constraint, ${ }^{27}$ can be written as

$$
A(t)=\mathbb{1}_{[-T / 2, T / 2]}(t)
$$

where $\mathbb{1}_{[\cdot, \cdot]}$ is the rectangle function. Although a constant amplitude windowing function maximises the overall energy of the pulse, it also introduces oscillations, known as Gibbs phenomena, ${ }^{28}$ in the Fourier domain.

Sonar systems do not have such a strong constraint, and more freedom is allowed in the pulse design. To keep more integrity in the Fourier domain, sonar pulses may use a Gaussian windowing such as

$$
A(t)=e^{-t^{2} / 2 \sigma^{2}} .
$$

The matched filter response $s_{f}(t)$ of the pulse [Eq. (8)] is given by

$$
\begin{aligned}
s_{f}(t) & =\int_{-\infty}^{+\infty} s^{*}\left(t^{\prime}\right) s\left(t^{\prime}+t\right) \mathrm{d} t^{\prime} \\
& =e^{2 i \pi\left(f_{0}+[\Delta f / 2 T] t\right) t} \int_{-\infty}^{+\infty} A\left(t^{\prime}\right) A\left(t^{\prime}+t\right) \exp \left[2 i \pi \frac{\Delta f}{T} t^{\prime} t\right] \mathrm{d} t^{\prime} .
\end{aligned}
$$

The computation of Eq. (11) with the windowing function [Eq. (9)] used for radar system gives the well-known solution $^{27}$

$$
s_{f}(t)=T \frac{\sin [\pi t \Delta f(1-|t| / T)]}{\pi t \Delta f} e^{2 i \pi f_{0} t} .
$$

Considering now the Gaussian windowing [Eq. (10)], Eq. (11) rewrites as

$$
\begin{aligned}
s_{f}(t)= & e^{2 i \pi\left(f_{0}+[\Delta f / 2 T] t\right) t} e^{-t / 2 \sigma^{2}} \\
& \times \int_{-\infty}^{+\infty} \exp \left[-\frac{t^{\prime 2}}{\sigma^{2}}+\left(-\frac{t}{\sigma^{2}}+2 i \pi \frac{\Delta f}{T} t\right) t^{\prime}\right] \mathrm{d} t^{\prime} .
\end{aligned}
$$

Using the Gaussian integral with complex offset result ${ }^{29}$ given by

$$
\int_{-\infty}^{+\infty} \exp \left(-a x^{2}+b x\right) \mathrm{d} x=\sqrt{\frac{\pi}{a}} e^{b^{2} / 4 a},
$$

for $a$ and $b \in \mathbb{C} /\{0\}$ within Eq. (13) leads to

$$
s_{f}(t)=\sigma \sqrt{\pi} \exp \left[-\left(\frac{1}{4 \sigma^{2}}+\pi^{2} \frac{\Delta f^{2}}{T^{2}} \sigma^{2}\right) t^{2}\right] e^{2 i \pi f_{0} t}
$$

To conclude, the matched filter response $s_{f}(t)$ can be written as

$$
s_{f}(t)=B(t) e^{2 i \pi f_{0} t}
$$

with

$$
\begin{aligned}
B(t)= & T \frac{\sin [\pi t \Delta f(1-|t| / T)]}{\pi t \Delta f} \\
& (\text { rectangular windowing }), \\
= & \sigma \sqrt{\pi} \exp \left[-\left(\frac{1}{4 \sigma^{2}}+\pi^{2} \frac{\Delta f^{2}}{T^{2}} \sigma^{2}\right) t^{2}\right]
\end{aligned}
$$

(Gaussian windowing).

\section{CSAS PSF}

As discussed previously, the CSAS response is computed by integrating the received echoes along the trajectory $\mathcal{C}$, as described in Eq. (4). The CSAS PSF is computed considering one normalised scatterer located in the centre $\mathbf{O}$. Exploiting the circular geometry of the problem, and within the neighbourhood of $\mathbf{O}$, the PSF $I=I(r)$, where $r$ is the distance to $\mathbf{O}$, and can be written as 


$$
I(r)=\int_{\theta=0}^{2 \pi} C(\theta) e^{4 i \pi f_{0} r \cos \theta / c} \mathrm{~d} \theta
$$

with $C(\theta)=B(2 r \cos \theta / c)$.

There are no general analytical solutions to Eq. (19). However, the only contributing terms of the MacLaurin series of $C(\theta)$ in Eq. (19) would be

$$
C(0)+\sum_{2 n=2}^{+\infty} \frac{C^{(2 n)}(0)}{2 n !} \theta^{2 n}
$$

Using a first order approximation of $C(\theta)$ into Eq. (19) then leads to the general CSAS PSF

$$
\begin{aligned}
I(r) & \approx C(0) \int_{\theta=0}^{2 \pi} e^{4 i \pi f_{0} r \cos \theta / c} \mathrm{~d} \theta \\
& =2 \pi C(0) J_{0}(2 k r),
\end{aligned}
$$

with $k=2 \pi f_{0} / c$ the wave number. $J_{0}(\cdot)$ represents the Bessel function of the first kind of order 0 .

For the next simulations, the central frequency $f_{0}$ of the CSAS system is $100 \mathrm{kHz}$, the bandwidth, $\Delta f$, is $20 \mathrm{kHz}$, and the pulse duration $T$ is $1 \mathrm{~ms}$. Figures 2(a) and 2(b) show a

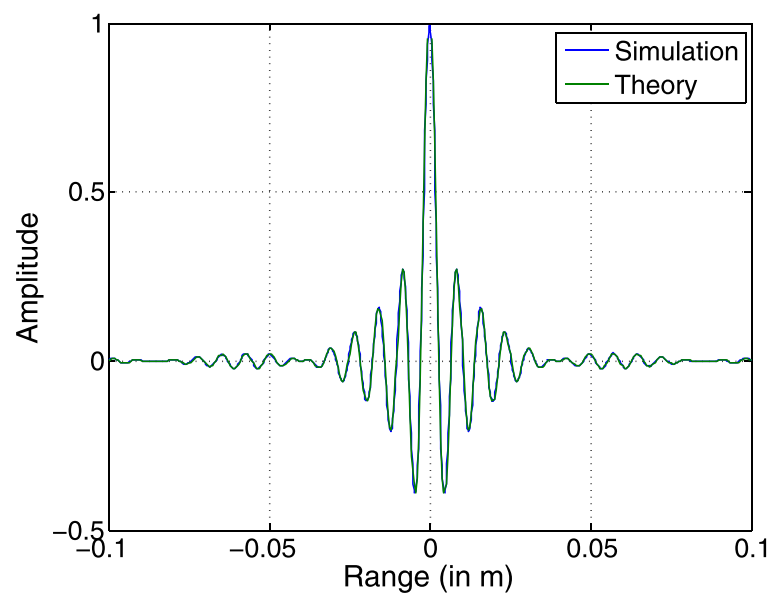

(a)

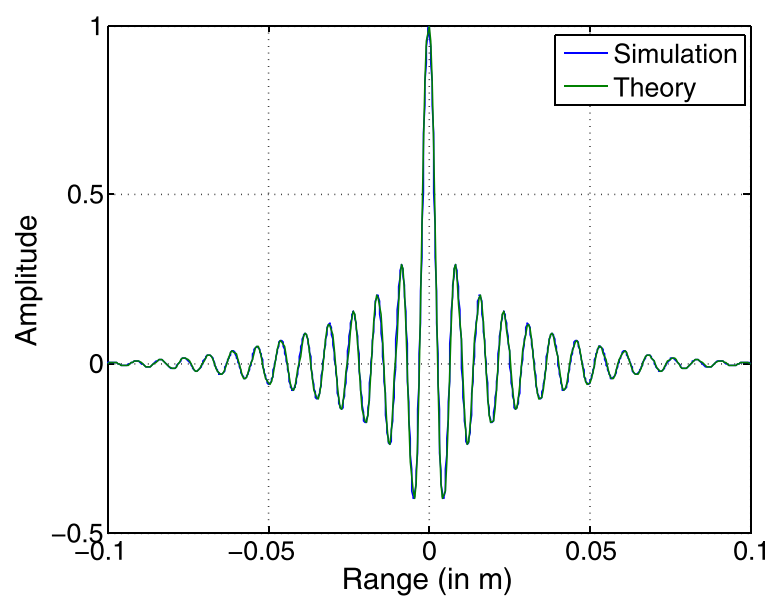

(b)

FIG. 2. (Color online) Comparison between the full SAS integration from Eq. (19) and the approximation from Eq. (21) for a pulse with (a) a rectangular window and (b) a Gaussian window. comparison between the simulated PSF computed numerically from Eq. (19) by integrating the matched filter response over the circular trajectory $\mathcal{C}$ and the approximation result of Eq. (21) for, respectively, a rectangular windowing and a Gaussian windowing. The great matching between the simulated data and Eq. (21) shows that the rest of the MacLaurin series does not contribute to a great extent to the CSAS PSF.

In Figs. 3(a) and 3(b), the resulting PSF image is drawn for a rectangular window and a Gaussian window, respectively. One interesting point is worth raising: while the rectangular windowing compresses the PSF energy more than the Gaussian windowing, it also introduces noticeable ringing effects, visible in Figs. 2(a) and 3(a), which extend radially more than the $B(t)$ of the Gaussian window. The tail distribution is heavier for the rectangular window than for the Gaussian window, introducing image artefacts which can be severe if they add constructively.

\section{Scatterer energy and resolution}

This section discusses the problem of resolution. A CSAS resolution criteria based on the energy leakage of the PSF is then proposed.

The classical definition for resolution is given by the formula: $c / 2 \Delta f$, and comes from the $-3 \mathrm{~dB}$ spatial width of the matched filter response for a rectangular window. ${ }^{27}$ However this formula is only a rough approximation and does not take into account the waveform design nor any

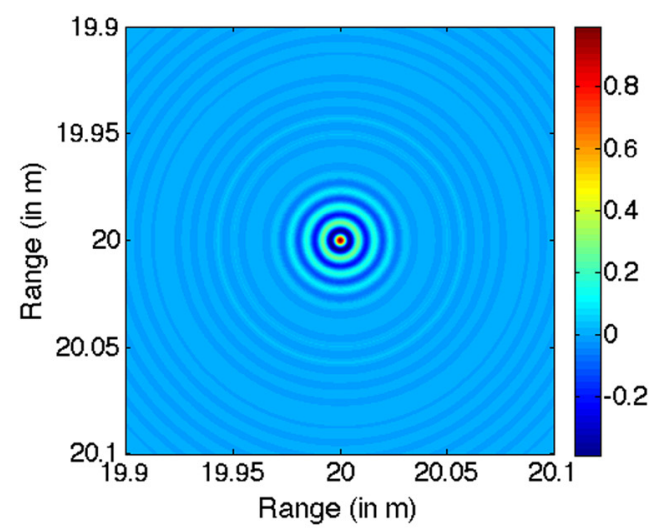

(a)

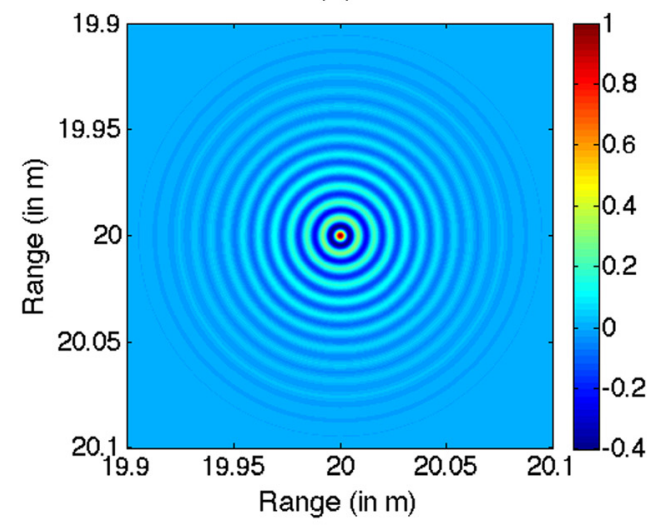

(b)

FIG. 3. (Color online) CSAS image of a scattering point using a pulse with (a) a rectangular window and (b) a Gaussian window. 
energy leakage contribution due to the imaging process. The resolution formula only focuses on point targets and does not reflect the resolution that can be achieved for imaging continuous interface like seabed in the SAS/CSAS case. In practice, the sidelobes of Eq. (21) can have a great impact on the final image, as observed in Figs. 3(a) and 3(b).

Because this paper focuses on the theoretical response of scattering points, it is interesting to look at the implications of the collapsing of the $\exp (\cdot)$ function from the matched filter response in Eq. (16) into the Bessel function $J_{0}(\cdot)$ in Eq. (21) and in particular the location of the first zero-crossing. Let $z_{0}$ be the first positive zero of the Bessel function $J_{0}(\cdot)$, i.e., $z_{0}=\min _{z>0}\left\{z \mid J_{0}(z)=0\right\}$. The width of the central peak for a CSAS system is then given by the width $2 z_{0}$ which corresponds spatially to $z_{0} c / 2 \pi f_{0}$. It is interesting to link this value to the same parameter for the matched filter [Eq. (16)]: $c / 4 f_{0}$. The ratio between the two (matched filter over CSAS), $\pi / 2 z_{0}$, is, first, independent of the central frequency, $f_{0}$, and the bandwidth, $\Delta f$, of the system, but most of all, this ratio is inferior to 1 . The degeneration of the dominant term $e^{2 i \pi f_{0}}$ of the matched filter response to a Bessel function, obtained during the CSAS processing, therefore increases the width of the central peak compared to the one obtained with matched filtering by around $50 \%$.

This section now links the CSAS resolution to the energy leakage of the PSF. The total energy $E$ of a normalised scatterer located in $\mathbf{O}$ is given by

$$
\begin{aligned}
E & =2 \pi \int_{r=0}^{+\infty} I^{*}(r) I(r) r \mathrm{~d} r \\
& =2 \pi \int_{r=0}^{+\infty}\left[B(2 r / c) J_{0}(2 k r)\right]^{2} r \mathrm{~d} r .
\end{aligned}
$$

A solution of Eq. (23) has been proposed by Lukin. ${ }^{30}$ In his paper, Lukin computes the total energy $P_{0}$ of a vortex Bessel-Gaussian beam $U_{0}(r, \phi)$ defined in Eq. (24),

$$
U_{0}(r, \phi)=E_{0} \exp \left(-\frac{r^{2}}{2 a_{0}^{2}}-\frac{i \gamma}{2 R_{0}} r^{2}\right) J_{m}(\beta r) \exp (i m \phi),
$$

where $J_{n}$ are the Bessel functions of the first kind for integer order $n$, by

$$
\begin{aligned}
P_{0} & =\int_{r=0}^{+\infty} \int_{\phi=0}^{2 \pi} U_{0}(r, \phi) U_{0}^{*}(r, \phi) r \mathrm{~d} r \mathrm{~d} \phi \\
& =\pi a_{0}^{2} E_{0}^{2} \exp \left(-\frac{\beta^{2} a_{0}^{2}}{2}\right) I_{0}\left(\frac{\beta^{2} a_{0}^{2}}{2}\right) .
\end{aligned}
$$

Equation (21) with a Gaussian window relates to Eq. (24) with the following substitutions:

$$
\begin{aligned}
& E_{0}=\sigma \sqrt{\pi}, \\
& a_{0}^{2}=\frac{1}{2} \frac{c^{2} \sigma^{2} T^{2}}{T^{2}+4 \pi^{2} \Delta f^{2} \sigma^{4}}, \\
& \beta=2 k,
\end{aligned}
$$

$$
\begin{aligned}
& m=0, \\
& \gamma=0 .
\end{aligned}
$$

For a sonar system with a quality factor $Q>2$, the product $\beta^{2} a_{0}^{2}$ is large $(\gg 1)$ and the asymptotic form of the Bessel function, $J_{0}(\cdot)$ leads to

$$
\begin{aligned}
e^{-z} I_{0}(z) & =e^{-z} J_{0}(i z) \approx e^{-z} \sqrt{\frac{2}{i \pi z}} \cos (i z-\pi / 4) \\
& =\frac{1}{\sqrt{2 \pi z}}\left(-i e^{-2 z}+1\right) \approx \frac{1}{\sqrt{2 \pi z}} .
\end{aligned}
$$

Using the result (31) within Eq. (25) leads finally to the total energy $E$ of the CSAS image of the PSF,

$$
E=\pi^{3 / 2} \sigma^{2} \frac{a_{0}}{\beta}
$$

To assess the CSAS resolution, one needs to calculate the radial diffusion $E\left(r_{0}\right)$ of the total energy $E$,

$$
E\left(r_{0}\right)=2 \pi \int_{r=0}^{r_{0}}\left[B(r) J_{0}(2 k r)\right]^{2} r \mathrm{~d} r .
$$

Obviously

$$
\lim _{r_{0} \rightarrow \infty} E\left(r_{0}\right)=E \text {. }
$$

Using the asymptotic form of the Bessel function $J_{0}(\cdot)$ and the fact that the variation of $B(r)^{2}$ is slow compared to the period of the sine, leads to

$$
\begin{aligned}
E\left(r_{0}\right) & \approx 2 \pi \int_{0}^{r_{0}} B(r)^{2} \frac{1}{\pi k r} \cos ^{2}(2 k r-\pi / 4) r \mathrm{~d} r \\
& =\frac{1}{k} \int_{0}^{r_{0}} B(r)^{2} \mathrm{~d} r+\frac{1}{k} \int_{0}^{r_{0}} B(r)^{2} \sin (4 k r) \mathrm{d} r \\
& \approx \frac{1}{k} \int_{0}^{r_{0}} B(r)^{2} \mathrm{~d} r .
\end{aligned}
$$

Considering the Gaussian windowing [Eq. (10)], the energy diffusion $E\left(r_{0}\right)$ finally becomes

$$
\begin{aligned}
E\left(r_{0}\right) & \approx \frac{\pi \sigma^{2}}{k} \int_{0}^{r_{0}} \exp \left(-\frac{r^{2}}{a_{0}^{2}}\right) \mathrm{d} r \\
& =\pi^{3 / 2} \sigma^{2} \frac{a_{0}}{\beta} \operatorname{erf}\left(a_{0} r_{0}\right) .
\end{aligned}
$$

Figure 4 plots the radial energy diffusion $E\left(r_{0}\right)$ using Eq. (33) and the proposed approximation [Eq. (38)] for a CSAS system with $f_{0}=100 \mathrm{kHz}, \Delta f=20 \mathrm{kHz}, T=1 \mathrm{~ms}$, $\sigma=2 \times 10^{-2}$. Equation (38) follows very closely the trend of Eq. (33) and respects the full energy value of Eq. (34). The only phenomena non simulated by Eq. (38) are the oscillations smoothed by the approximation [Eq. (37)].

From the energy diffusion expression, it is then possible to define the CSAS resolution as a percentage of the total energy of the PSF. Note that that percentage is easily 


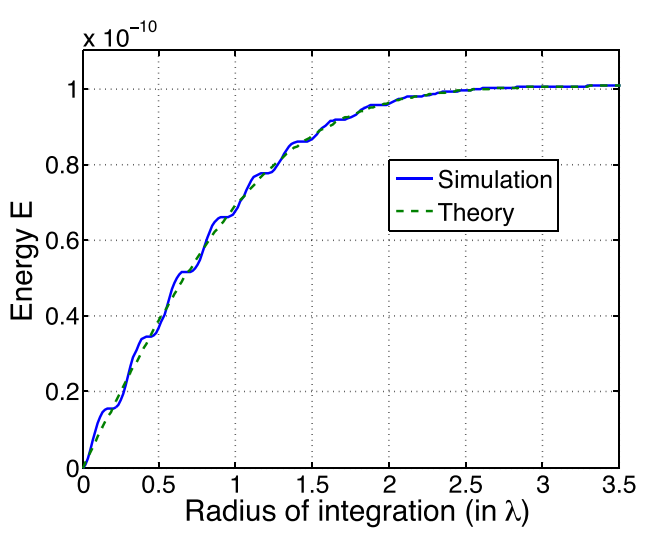

FIG. 4. (Color online) $E\left(r_{0}\right)$ energy diffusion factor in a function of $\lambda$ computed with full PSF from Eq. (19) and compared with the approximation [Eq. (38)], with $f_{0}=100 \mathrm{kHz}, \Delta f=20 \mathrm{kHz}, T=1 \mathrm{~ms}, \sigma=2 \times 10^{-2}$.

computed by $E\left(r_{0}\right) / E=\operatorname{erf}\left(a_{0} r_{0}\right)$. As an example, for the parameters used to compute Fig. 4, a resolution cell based on $50 \%$ of the PSF energy would be a circle with a radius of approximately $0.75 \lambda$. For a resolution based on $90 \%$ of the energy, the resolution cell radius increases to around $1.75 \lambda$.

\section{DECONVOLUTION}

This section proposes a thresholding based deconvolution using adapted wave atoms in order to increase the CSAS resolution. But first, the Fourier transform of the PSF [Eq. (21)] needs to be calculated.

\section{A. Fourier domain}

In polar coordinates, the Fourier transform $F(\rho, \phi)$ of a function $f(r, \theta)$ can be computed as follows:

$$
F(\rho, \phi)=\int_{r=0}^{+\infty} \int_{\theta=0}^{2 \pi} f(r, \theta) e^{2 i \pi \rho r \cos (\theta-\phi)} r \mathrm{~d} r \mathrm{~d} \theta .
$$

For a circularly symmetric function, i.e., $f(r, \theta)=f(r)$, the Fourier transform of $f(r)$ is also circularly symmetric and $F(\rho, \phi)=F(\rho)$, and $F(\rho)$ reduces to

$$
F(\rho)=2 \pi \int_{r=0}^{+\infty} f(r) J_{0}(2 \pi r \rho) r \mathrm{~d} r .
$$

Applying formula (40) to Eq. (21) gives for the Gaussian windowing case

$$
\begin{aligned}
\hat{I}(\rho)= & 4 \pi^{5 / 2} \sigma \int_{r=0}^{+\infty} \exp \left(-\frac{r^{2}}{2 a_{0}^{2}}\right) J_{0}(2 k r) J_{0}(2 \pi r \rho) r \mathrm{~d} r \\
= & 8 \pi^{5 / 2} \sigma a_{0}^{2} \int_{r=0}^{+\infty} \exp \left(-r^{2}\right) J_{0}\left(2 \sqrt{2} k a_{0} r\right) \\
& \times J_{0}\left(2 \sqrt{2} \pi a_{0} \rho r\right) r \mathrm{~d} r
\end{aligned}
$$

The generalisation of Weber's second exponential integral ${ }^{31}$ gives for any $(a, b) \in \mathbb{R}^{2}$,

$$
\int_{0}^{+\infty} J_{0}(a x) J_{0}(b x) e^{-x^{2}} x \mathrm{~d} x=\frac{1}{2} I_{0}\left(\frac{a b}{2}\right) \exp \left(-\frac{a^{2}+b^{2}}{4}\right)
$$

where $I_{0}(\cdot)$ is the modified Bessel function of the first kind. Using relation (42) into Eq. (41) gives the analytical expression of the Fourier transform of the PSF,

$$
\hat{I}(\rho)=4 \pi^{5 / 2} \sigma a_{0}^{2} I_{0}\left(4 \pi k a_{0}^{2} \rho\right) \exp \left[-2 a_{0}^{2}\left(k^{2}+\pi^{2} \rho^{2}\right)\right] .
$$

Using the modified Bessel asymptotic form, $I_{0}(z) \approx e^{z} /$ $\sqrt{2 \pi z}$, into Eq. (43) finally leads to

$$
\hat{I}(\rho) \approx 2 \pi^{3 / 2} \frac{\sigma a_{0}}{\sqrt{2 k \rho}} \exp \left[-2 a_{0}^{2}(\pi \rho-k)^{2}\right] .
$$

Similar to Eq. (32), the use of the asymptotic formula for $I_{0}(\cdot)$ is justified for a sonar system with a quality factor $Q>2$. Equation (44) demonstrates that, in the Fourier domain, the CSAS image energy is concentrated in a ring whose mean diameter is $2 f_{0} / c$ with a variance of $1 / \pi^{2} a_{0}^{2}$. This property is pictured in Fig. 5(a) where the twodimensional (2D) Fourier transform of the PSF of the CSAS processing has been computed using the same parameters as the ones in Sec. II. Figure 5(b) shows the great agreement between the numerical result given by the fast Fourier transform of Eq. (19) and the approximation (44) of the radial component of the Fourier transform $\hat{I}(\rho)$.

\section{B. Deconvolution}

The deconvolution problem is a very well known illposed problem found, for example, in image processing ${ }^{32}$ or optics. ${ }^{33}$ The resulting CSAS image $J$ after synthetic aperture processing can be expressed as

$$
J=I * S_{s}+w,
$$

where $S_{s}$ represents the seafloor scattering intensity and the parameter to be recovered and $w$ is the noise introduced to the system. According to the sampling scheme presented in Sec. II A, $I$ is independent of the location on which $J$ is computed. Sections IIC and III A give the analytical expressions of $I$ both in the image and Fourier domain, the resolution of Eq. (45) therefore falls into the classical deconvolution problem where the convolution kernel is uniform and known. It is worth pointing out that, for classic deconvolution problems in optics related domains (such as optical microscope or telescope), the PSF often models a blur, introduced for example by motion or lens aberration/defects, and it usually acts as a low frequency filter. For the current problem of CSAS imaging and according to the results from Eq. (44), the PSF acts like a bandpass filter.

Traditional approaches to the deconvolution problem involves the Fourier transform of Eq. (45),

$$
\hat{J}=\hat{I} \hat{S}_{s}+\hat{w}
$$




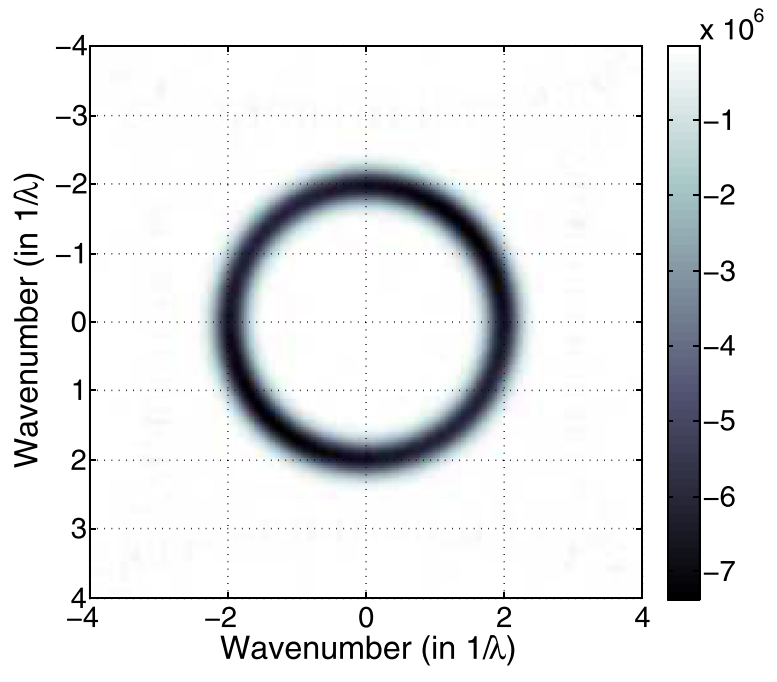

(a)

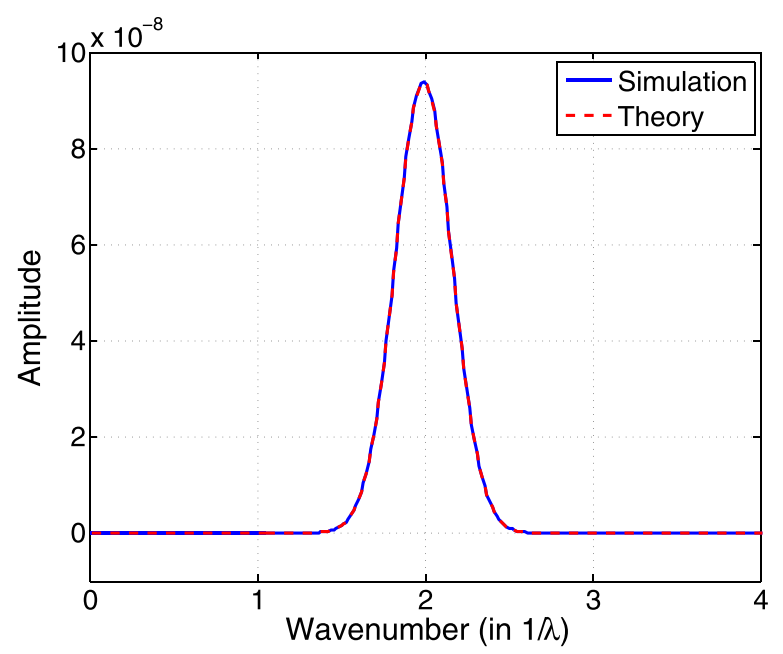

(b)

FIG. 5. (Color online) (a) 2D Fourier transform of the impulse response of a CSAS. (b) Comparison between simulated and theoretical [given by Eq. (44)] computation of the radial component $\hat{I}(\rho)$ of the Fourier transform of the impulse response of a CSAS image.

where the operator $\hat{x}$ denotes the Fourier transform. A naive solution to Eq. (45) would be $\hat{I}^{-1} \hat{J}$, where $\hat{I}^{-1}$ is defined as $\hat{I}^{-1}=1 / \hat{I}$ if $|\hat{I}| \geq \eta$, or $\hat{I}^{-1}=0$ if $|\hat{I}|<\eta$. $\eta$ is a given threshold depending on the noise level. The drawback of the naive approach lies in the fact that the noise is artificially boosted for low values of $\hat{I}$. Deconvolution can also be performed thanks to the Wiener deconvolution filter $\hat{G}$ defined as

$$
\hat{G}=\frac{\hat{I}^{*} \hat{s}}{|\hat{I}|^{2} \hat{s}+\hat{n}},
$$

where $\hat{s}$ and $\hat{n}$ are, respectively, the mean power spectral density of $S_{s}$ and $w$. The result is then given in the Fourier domain by $\hat{G} \hat{J}$.

As suggested by Kalifa et al. in Ref. 34, deconvolution can also be done by decomposing $J * I^{-1}$ into an orthogonal basis $\mathcal{B}=\left\{\psi_{n}\right\}_{n \in[1, N]}$, and thresholding the resulting coefficients $\left\langle J * I^{-1}, \psi_{n}\right\rangle$. The aim of this section is to propose a basis $\mathcal{B}$ which concentrates most efficiently the energy of the signal $J * I^{-1}$. The mirror wavelet basis proposed by Kalifa et $a l .{ }^{34}$ allows a finer resolution at high frequencies, and can then restore more accurately high frequency image phenomena such as sharp transitions. Given the current problem and, in particular, the CSAS PSF energy distribution, wavelets or mirror wavelets do not divide the Fourier space in an optimal manner. Modified wavelet bases have been proposed to better take into account particular directionality or circular geometry in the Fourier space. Such methods includes the curvelets introduced by Candes and Donoho in Ref. 35 or wave atoms proposed by Demanet and Ying in Ref. 36.

As Sec. III A demonstrates, the CSAS image energy is concentrated in a ring with a diameter and variance entirely determined by the SAS pulse parameters. This result leads naturally to the construction of the wave atoms $\left\{\psi_{n}\right\}_{n}$ adapted to the PSF expressed in Eq. (21). The wave atoms $\psi_{n}$ are constructed as follows:

$$
\psi_{n}(x, y)=B\left(\frac{2 r}{c}\right) J_{0}\left[2 k\left(x \cos \theta_{n}-y \sin \theta_{n}\right)\right],
$$

where $r=\sqrt{x^{2}+y^{2}}$, and $\theta_{n}$ is the directionality of the wave atom $\psi_{n}$. Figure 6(a) shows an example of a wave atom $\psi_{n}$ for the pulse parameters previously used and an angle of $\theta_{n}=45^{\circ}$. Figure $6(\mathrm{~b})$ shows a representation of the distribution of $\left\{\psi_{n}\right\}_{n \in[1, N]}$ in the Fourier space. Each circle represents the location of one wave atom $\psi_{n}$ for one particular angle.

Note that, as defined, $\mathcal{B}=\left\{\psi_{n}\right\}_{n \in[1, N]}$ is not strictly speaking a basis for the simple reason that the full collection of these wave atoms do not cover the entire Fourier space. However, completing $\mathcal{B}$ is not needed for the current purpose because the Fourier space not covered by $\left\{\psi_{n}\right\}_{n \in[1, N]}$ is null for a CSAS image as demonstrated in Sec. III A. Reducing the analysis of $\mathcal{B}$ then automatically solves the thresholding problem. The estimated image $\tilde{S}_{S}$ is then given by

$$
\tilde{S}_{s}=\sum_{n=1}^{N}\left\langle J * I^{-1}, \psi_{n}\right\rangle \psi_{n}
$$

As an example, results are given for a four scatterer target. Each scatterer is located on one corner of a square whose size is $\lambda$, the top-left scatterer of the square target is located at $[20 \mathrm{~m}, 20 \mathrm{~m}]$. Again, the same set of parameters $\left(f_{0}=100 \mathrm{kHz}\right.$,

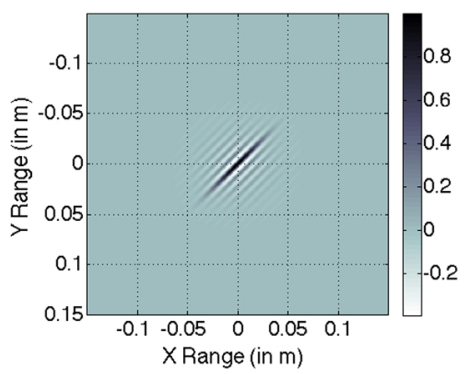

(a)

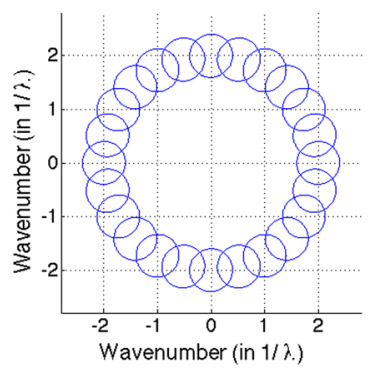

(b)
FIG. 6. (Color online) (a) Example of an adapted wave atom $\psi_{n}$ for a direction of $45^{\circ}$ and (b) representation of the distribution of $\mathcal{B}=\left\{\psi_{n}\right\}_{n \in[1, N]}$ in the Fourier domain. 
$\Delta f=20 \mathrm{kHz}, T=1 \mathrm{~ms}$, and $\sigma=2 \times 10^{-2}$ ) are used for this simulation. The SAS system follows a circular trajectory centred in $[0 \mathrm{~m}, 0 \mathrm{~m}]$ with a radius of $40 \sqrt{2} \mathrm{~m}$. The virtual CSAS echoes, computed for a virtual centre point [20 m, $20 \mathrm{~m}]$ are then computed using the sampling scheme Eq. (7). The virtual CSAS then follows a circle centred in $[20 \mathrm{~m}, 20 \mathrm{~m}]$ with a radius of $20 \mathrm{~m}$ as pictured in Fig. 7(a).

As mentioned in Sec. II A, a radially displaced scattering point is not sampled uniformly in view angles. Figure 7(b) displays the number of views density for two scattering points located, respectively, at $[20 \mathrm{~m}, 20 \mathrm{~m}]$ and $[0 \mathrm{~m}, 0 \mathrm{~m}]$. As expected the centred scatterer exhibits a uniform density, while the offset scattering point shows great variations in the number of views density. At $45^{\circ}$, the SAS system is the closest to the offset scatterer, and as a consequence the number of view density is the lowest. The region around the $45^{\circ}$ view will then have the lowest contribution in the formation of the CSAS image. The real parts of the CSAS images of the two scatterers differ only slightly from each other (in the order of $1 \%$ ), however their 2D Fourier transforms can exhibit significant differences. Figure 7(c) displays the 2D Fourier transform of the radially displaced scatterer. Compared to Fig. 5(a), the spectra are not angularly uniform. The angular intensity of the spectra varies accordingly to the number of views density with $+\pi / 2$ angle shift from the spatial plane to the Fourier plane. When the resampling scheme Eq. (7) is applied, however, the spectra, as shown in Fig. 7(d), became uniform and consistent with the results derived in Sec. III A.
Considering now the four scatterer target described earlier, Fig. 8(a) pictures the target raw CSAS image using the circular back-projection algorithm. Due to the relatively low bandwidth of the system and the close locations of the target scatterers, a strong energy leakage as well as strong interferences are observed in Fig. 8(a). Figures 8(b) and 8(c) picture the results obtained, respectively, by the naive deconvolution algorithm and the Wiener deconvolution filter [Eq. (47)] with $30 \mathrm{~dB}$ signal-to-noise ratio. As expected, the deconvolution process greatly amplifies the noise.

Figure 8(d) shows the result of the deconvolution estimator using a basis of 64 wave atoms as described in Eq. (48). Note that the upper bound of the number of admissible wave atoms depends on the grid resolution on which the back propagation is processed. In their paper, Demanet and Ying $^{36}$ compute this number as the admissible titles in the frequency plane as a function of the atom wave scale. The proposed atom wave deconvolution method increases the resolution as defined in Sec. II D, and reduces drastically the energy leakage. Figure 9 illustrates specifically this point by drawing, similar to Fig. 4, the energy diffusion of one scatterer as a function of $\lambda$ for, respectively, the raw CSAS PSF and the atom wave deconvolution PSF. With the wave atom deconvolution, $80 \%$ and $95 \%$ of the energy of a scattering point are concentrated in a circle of, respectively, $0.25 \lambda$ and $0.75 \lambda$ radius. Circles of, respectively, $1.3 \lambda$ and $2 \lambda$ are necessary to concentrate the same amount of energy for the raw CSAS response. This result shows that the adapted wave atom deconvolution algorithm concentrates efficiently the

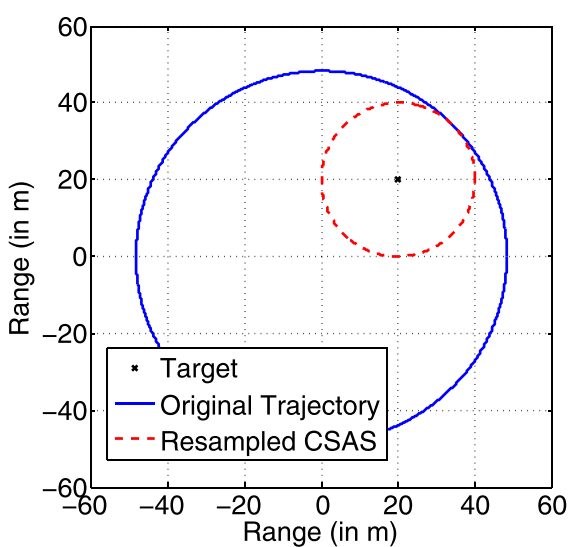

(a)

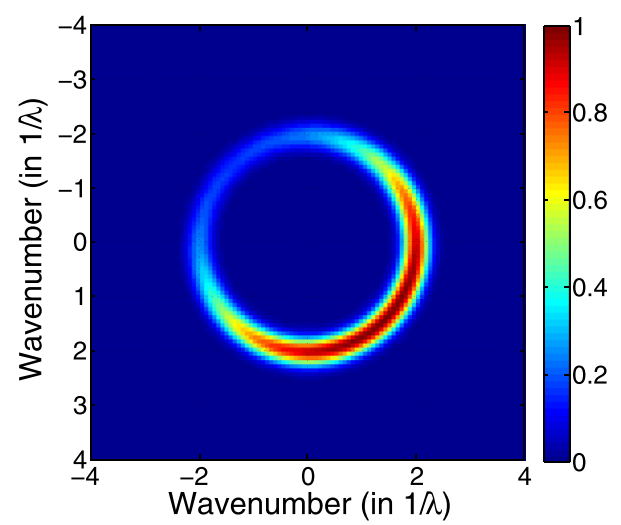

(c)

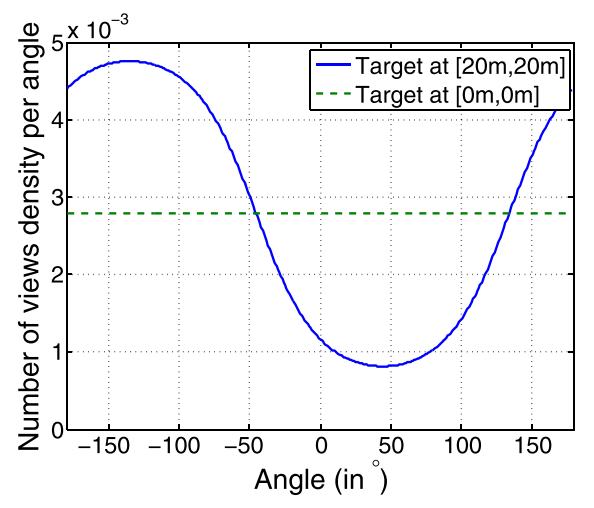

(b)

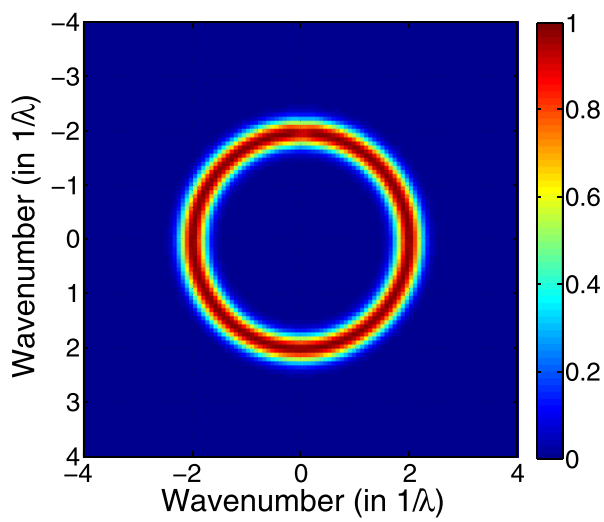

(d)
FIG. 7. (Color online) (a) CSAS resampling equivalent of a target located at [20 m, $20 \mathrm{~m}]$. (b) Number of views density for a target located at [20 m, 20 m] and a centred target. (c) Normalised 2D Fourier transform for a scattering point target located at $[20 \mathrm{~m}$, $20 \mathrm{~m}$ ] performed without the resampling scheme. (d) Performed with the resampling scheme Eq. (7). 


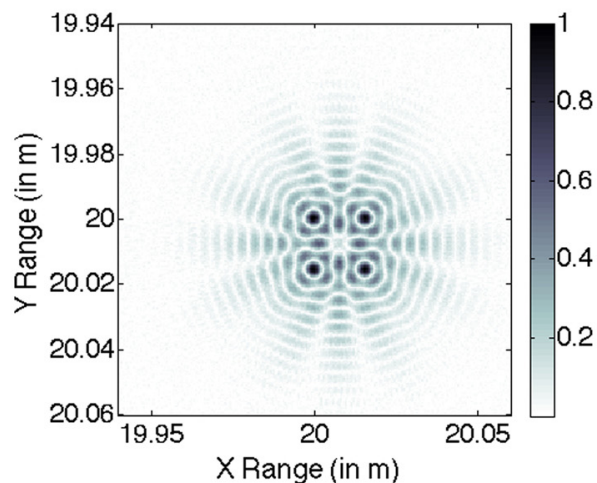

(a)

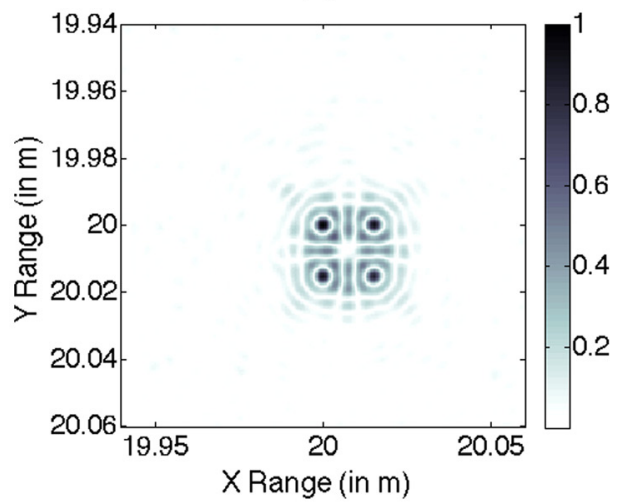

(c)

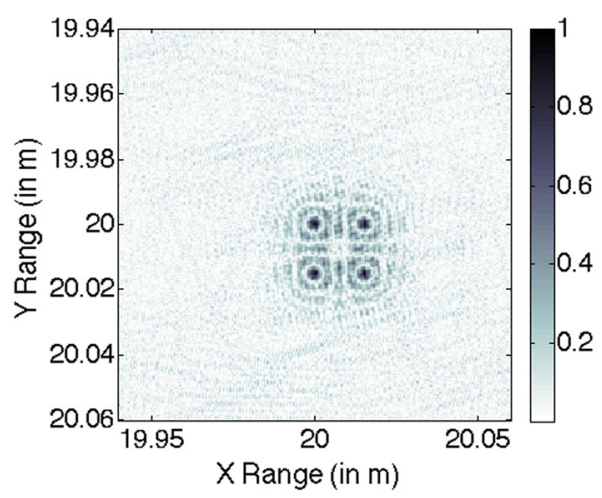

(b)

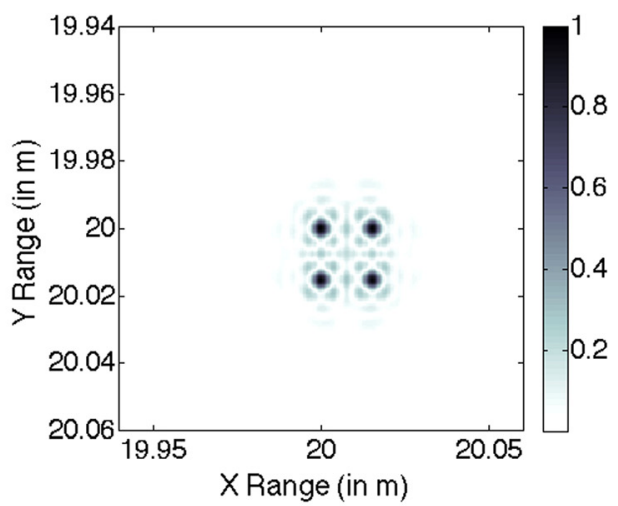

(d)
FIG. 8. (Color online) (a) Original CSAS image of a four scattering point target. (b) deconvolution, (c) Wiener deconvolution, and (d) adapted wave atoms Naive deconvolution. scatterer energy. The disadvantage of this method is the introduction of noise in the CSAS image. Figure 9 shows that around $1 \%$ of the scatterer energy is dispersed in the scattering point neighbourhood. It is interesting to note that the Wiener deconvolution also exhibits a sharp increase of the normalized energy for small radius of integration, as shown in Fig. 9, and then tends to concentrate the PSF energy. The level of noise introduced by the filter however is highly significant and degrades greatly the overall image quality. The slow rising energy for the Wiener deconvolution in Fig. 9 demonstrates this point: mostly noise energy is integrated after $1.5 \lambda$ integration radius.

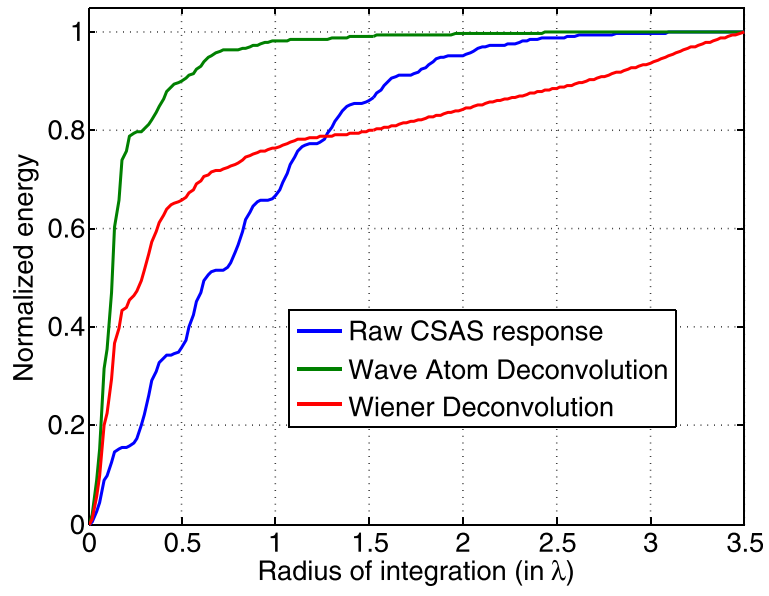

FIG. 9. (Color online) Normalised energy diffusion of the PSF in a function of the radius of integration expressed in $\lambda$ for the raw CSAS PSF and the deconvoluted PSF.
As demonstrated in Sec. II D, the resolution is highly dependent upon the windowing function applied to the pulse. This paper presents the analytical results for Gaussian and rectangular windowing. Other windowing functions are also used in sonar systems such as Tukey or Taylor weighting windows which compromise between output energy and ringing control. The PSF for these functions is not analytically tractable, and has to be computed numerically. In more general terms, the PSF and resolution computations of a specific sonar system would have to take into account, besides the windowing function of the pulse, the calibrated transducers and amplifiers responses. Finally, the transmitter and receiver beampattern will have an impact on the PSF and will also have to be taken into account in the computation. One solution to this problem may be to compensate for the beampattern assuming that the impact on the noise level will be minimal.

\section{CONCLUSIONS}

This paper lays down the theoretical background for CSAS processing by proposing the closed-form solutions of the CSAS PSF in the image domain and the Fourier domain. The resolution problem has been reformulated to integrate an energy diffusion approach. Thanks to the analytical formulation of the CSAS PSF, an adapted atom wave basis has been built to match the CSAS problem, and a thresholding deconvolution, using this specific adapted basis, has been proposed. The suggested algorithm shows encouraging results for relatively narrowband SAS systems. Although only simulated results are presented here, future works will 
include tests of the proposed deconvolution method on real CSAS data, where the algorithm will have to cope with realistic noise and continuous seabed interface. Further development also includes the extension of the CSAS imaging problem to more realistic 3D scene geometry. It is also worth noting that, although the simulations presented here have focused on sonar synthetic aperture systems, the theory proposed should apply to similar radar systems equally well.

\section{ACKNOWLEDGMENTS}

This work was supported by the Engineering and Physical Sciences Research Council (EPSRC) Grant No. EP/ J015180/1 and the MOD University Defence Research Collaboration in Signal Processing.

${ }^{1}$ A. Bellettini and M. A. Pinto, "Theoretical accuracy of synthetic aperture sonar micronavigation using a displaced phase-center antenna," IEEE J. Ocean. Eng. 27(4), 780-789 (2002).

${ }^{2}$ M. Pinto, "Design of synthetic aperture sonar systems for high-resolution seabed imaging," Technical report, NURC-PR-2006-029 (2006).

${ }^{3}$ R. E. Hansen, T. O. Sæbø, H. J. Callow, and P. E. Hagen, "The SENSOTEK Synthetic Aperture Sonar: Results from HUGIN AUV trials," Technical report, Norwegian Defence Research Establishment (FFI) (2007).

${ }^{4}$ D. Shea, D. Dawe, J. Dillon, and S. Chapman, "Real-time SAS processing for high-arctic AUV surveys," in IEEE/OES Autonomous Underwater Vehicles $(A U V)$, Institute of Electrical and Electronics Engineers, Piscataway, NJ (October 6-9, 2014), pp. 1-5.

${ }^{5}$ F. Jean, "Shadows, a new synthetic aperture sonar system, by IXSEA SAS," in OCEANS 2006 - Asia Pacific, Institute of Electrical and Electronics Engineers, Piscataway, NJ (May 16-19, 2006), pp. 1-5.

${ }^{6}$ S. G. Kargl, K. L. Williams, and E. I. Thorsos, "Synthetic aperture sonar imaging of simple finite targets," IEEE J. Ocean. Eng. 37(3), 516-532 (2012).

${ }^{7}$ D. P. Williams and E. Fakiris, "Exploiting environmental information for improved underwater target classification in sonar imagery," IEEE Trans. Geosci. Remote Sens. 52(10), 6284-6297 (2014).

${ }^{8}$ J. Sawas, Y. Petillot, and Y. Pailhas, "Cascade of boosted classifiers for rapid detection of underwater objects," in Proceedings of the European Conference on Underwater Acoustics, Institute of Acoustics, St. Alban, United Kingdom (2010), pp. 1507-1516.

${ }^{9}$ Y. Pailhas, Y. Petillot, and C. Capus, "High-resolution sonars: What resolution do we need for target recognition?," EURASIP J. Adv. Signal Process. 2010(1), 205095 (2010).

${ }^{10}$ O. Daniell, Y. Petillot, S. Reed, J. Vazquez, and A. Frau, "Reducing false alarms in automated target recognition using local sea-floor characteristics," in Sensor Signal Processing for Defence (SSPD), Institute of Electrical and Electronics Engineers, Piscataway, NJ (September 8-9, 2014), pp. 1-5.

${ }^{11}$ P. Y. Mignotte, E. Coiras, H. Rohou, Y. Petillot, J. Bell, and K. Lebart, "Adaptive fusion framework based on augmented reality training," IET Radar, Sonar Navigation 2(2), 146-154 (2008).

${ }^{12}$ D. P. Williams and A. J. Hunter, "Multi-look processing of highresolution SAS data for improved target detection performance," in IEEE International Conference on Image Processing (ICIP), Institute of Electrical and Electronics Engineers, Piscataway, NJ (September 27-30, 2015), pp. 153-157.

${ }^{13}$ Y. Pailhas, C. Capus, and K. Brown, "Strategies for rapid target reacquisition: Principles and theoretical advantages," in Proceedings of the Institute of Acoustics, International Conference on Detection and
Classification of Underwater Targets, Institute of Acoustics, St. Alban, United Kingdom (2007), pp. 1-5.

${ }^{14}$ P. Patron, Y. Pailhas, J. Cartwright, F. Maurelli, Y. Petillot, J. Sawas, and N. Valeyrie, "Fully integrated multi-vehicles mine countermeasure missions," in 4th International Conference and Exhibition on Underwater Acoustic Measurements: Technologies \& Results (UAM 2011), Institute of Acoustics, St. Alban, United Kingdom (2011), pp. 159-166.

${ }^{15}$ M. Soumekh, "Reconnaissance with slant plane circular SAR imaging," IEEE Trans. Image Process. 5(8), 1252-1265 (1996).

${ }^{16}$ T.-K. Chan, Y. Kuga, and A. Ishimaru, "Experimental studies on circular sar imaging in clutter using angular correlation function technique," IEEE Trans. Geosci. Remote Sens. 37(5), 2192-2197 (1999).

${ }^{17}$ M. Pinheiro, P. Prats, R. Scheiber, M. Nannini, and A. Reigber, "Tomographic 3D reconstruction from airborne circular SAR," in IEEE International Geoscience and Remote Sensing Symposium (July 2009), Vol. 3, pp. III-21-III-24.

${ }^{18}$ H. Rudolf, D. Tarchi, and A. J. Sieber, "Combination of linear and circular SAR for 3D features," in Geoscience and Remote Sensing, IGARSS'97, Institute of Electrical and Electronics Engineers, Piscataway, NJ (August 1997), Vol. 4, pp. 1551-1553.

${ }^{19}$ J. B. Poisson, H. M. Oriot, and F. Tupin, "Ground moving target trajectory reconstruction in single-channel circular SAR," IEEE Trans. Geosci. Remote Sens. 53(4), 1976-1984 (2015).

${ }^{20}$ E. Ertin, R. L. Moses, and L. C. Potter, "Interferometric methods for three-dimensional target reconstruction with multipass circular SAR," IET Radar, Sonar Navigation 4(3), 464-473 (2010).

${ }^{21}$ B. G. Ferguson and R. J. Wyber, "Application of acoustic reflection tomography to sonar imaging," J. Acoust. Soc. Am. 117(5), 2915-2928 (2005).

${ }^{22}$ R. E. Hansen, T. O. Sæsbø, and H. J. Callow, "Circular synthetic aperture sonar results from autonomous underwater vehicle trials," J. Acoust. Soc. Am. 123(5), 3899-3899 (2008).

${ }^{23}$ S. K. Mitchell, K. N. Scarbrough, S. P. Pitt, and T. L. Kooij, High Resolution Circular SAS with Controlled Focus, 5th ed. (Institute of Acoustics, St. Alban, UK, 2006), Vol. 28, pp. 65-71.

${ }^{24}$ T. M. Marston, J. L. Kennedy, and P. L. Marston, "Autofocusing circular synthetic aperture sonar imagery using phase corrections modeled as generalized cones," J. Acoust. Soc. Am. 136(2), 614-622 (2014).

${ }^{25}$ T. M. Marston and J. L. Kennedy, "Volumetric acoustic imaging via circular multipass aperture synthesis," IEEE J. Ocean. Eng. 41, 852-867 (2016).

${ }^{26}$ D. S. Plotnick, P. L. Marston, and T. M. Marston, "Fast nearfield to farfield conversion algorithm for circular synthetic aperture sonar," J. Acoust. Soc. Am. 136(2), EL61-EL66 (2014).

${ }^{27} \mathrm{M}$. Cheney and B. Borden, Fundamentals of Radar Imaging (Society for Industrial and Applied Mathematics, Philadelphia, PA, 2008), 149 pp.

${ }^{28}$ J. W. Gibbs, "Fourier's series," Nature 59, 200 (1898).

${ }^{29}$ A. Zee, "Quantum field theory in a nutshell," in Nutshell Handbook (Princeton University Press, Princeton, NJ, 2003).

${ }^{30}$ I. P. Lukin, "Integral momenta of vortex Bessel-Gaussian beams in turbulent atmosphere," Appl. Opt. 55(12), B61-B66 (2016).

${ }^{31} \mathrm{G}$. N. Watson, Theory of Bessel Functions (Cambridge University Press, Cambridge, UK, 1966), 805 pp.

${ }^{32}$ R. H. T. Bates and M. J. McDonnell, Image Restoration and Reconstruction (Clarendon Press Oxford, Oxford, UK, 1986), 288 pp.

${ }^{33}$ J.-B. Sibarita, "Deconvolution microscopy," in Microscopy Techniques (Springer, New York, 2005), pp. 201-243.

${ }^{34} \mathrm{~J}$. Kalifa, S. Mallat, and B. Rougé, "Deconvolution by thresholding in mirror wavelet bases," IEEE Trans. Image Process. 12(4), 446-457 (2003).

${ }^{35}$ E. J. Candes and D. L. Donoho, "Continuous curvelet transform," Appl. Comput. Harmonic Anal. 19(2), 198-222 (2005).

${ }^{36} \mathrm{~L}$. Demanet and L. Ying, "Wave atoms and sparsity of oscillatory patterns," Appl. Comput. Harmonic Anal. 23(3), 368-387 (2007). 